\title{
Patient-Specific Simulations of Deep Brain Stimulation Electric Field with Aid of In-house Software ELMA
}

J ohannes D J ohansson, Fabiola Alonso and Karin Wårdell

The self-archived postprint version of this journal article is available at Linköping University Institutional Repository (DiVA):

http:/ / urn.kb.se/ resolve?urn=urn:nbn:se:liu:diva-162290

N.B.: When citing this work, cite the original publication.

J ohansson, J . D, Alonso, F., Wårdell, K., (2019), Patient-Specific Simulations of Deep Brain Stimulation Electric Field with Aid of In-house Software ELMA, 2019 41st Annual International Conference of the IEEE Engineering in Medicine and Biology Society (EMBC).

https:// doi.org/ 10.1109/EMBC.2019.8856307

Original publication available at:

https:/ / doi.org/ 10.1109/ EMBC.2019.8856307

Copyright: IEEE

http:// www.ieee.org/

(C) 2019 IEEE. Personal use of this material is permitted. However, permission to reprint/ republish this material for advertising or promotional purposes or for creating new collective works for resale or redistribution to servers or lists, or to reuse any copyrighted component of this work in other works must be obtained from the IEEE. 


\section{(C)2019 IEEE}

Cite as:

J. D. Johansson, F. Alonso, and K. Wårdell, "Patientspecific Simulations of Deep Brain Stimulation Electric Field with Aid of In-house Software ELMA " in the 41st International Engineering in Medicine and Biology Conference, Berlin, 2019, DOI: $10.1109 /$ EMBC. 2019.8856307 https://ieeexplore.ieee.org/document/8856307. 


\title{
Patient-Specific Simulations of Deep Brain Stimulation Electric Field with Aid of In-house Software ELMA
}

\author{
Johannes D. Johansson, Fabiola Alonso, and Karin Wårdell, Member IEEE
}

\begin{abstract}
Deep brain stimulation (DBS) is an established technique for reduction of symptoms in movement disorders. Finite element method (FEM) simulations of the electric field magnitude (EF) are useful for estimating the affected tissue around the DBS lead and this can help optimize the therapy. This paper describes how patient-specific FEM models can be set up with the aid of the Matlab-based in-house software tool ELMA. Electrode placement is determined from two coordinates in postoperative medical imaging and electric conductivity is assigned from preoperative magnetic resonance imaging (MRI) and patient-specific DBS data. Simulations are performed using the equation for steady currents in Comsol Multiphysics (CM). The simulated EF is superimposed on the preoperative MRI for evaluation of affected structures. The method is demonstrated with patient-specific simulations in the zona incerta and a globus pallidus example containing cysts with higher conductive which causes considerable distortion of the EF. The improved software modules and precise lead positioning simplifies and reduces the time for DBS EF modelling and simulation.
\end{abstract}

\section{INTRODUCTION}

DBS is an established technique for symptomatic treatment of movement disorders such as Parkinson's disease (PD), essential tremor (ET) and dystonia [1-3]. It is also being evaluated for a number of new indications, e.g. psychiatric conditions such as obsessive-compulsive disorder, major depression and Tourette syndrome [4-6]. The fundamental function of DBS is to disrupt the pathological neural activity in tissue surrounding the electrodes. However, the precise physiological mechanisms through which DBS relieves the symptoms of neurological disorders are not fully understood.

FEM simulations compared with clinical experimental studies [7, 8] have been used to estimate pulse widthdependent EF thresholds for tissue activation. Clinical investigations based on the EF extent compared to the surrounding anatomy, aim to aid the knowledge of DBS function and assist clinicians to customize the stimulation parameter settings for each patient. Such studies have been made for e.g. PD [9], ET [10] and Tourette syndrome [11].

FEM models have progressed from representing the brain as a homogenous conductor medium [12-14] to more realistic models, based on individual brain images. Various patientspecific modelling, simulation and visualization concepts where the heterogeneity of the brain is taken into account have been presented [15]. Our concept at the Department of

*Research supported by the Swedish Foundation for Strategic Research (Project BD15-0032), the Swedish Research Council (Vetenskapsrådet, Dnr. 2016-03564), and the Knut and Alice Wallenberg Foundation (Project Seeing Organ Function).
Biomedical Engineering at Linköping University is based on preoperative MRI to classify tissue for creation of a brain model consisting of electrical conductivities. The patient's own DBS settings are used to set up FEM simulations, and these are used to visualize the EF isolevels superimposed on the patient's preoperative MRI. This principle concept was first presented by Åström et al. in 2009 [16]. To simplify the simulation process, a first version of software module, ELMA 1.0, for creating the brain model was introduced [17]. The Linköping concept has since then been used in numerous studies [10,11, 18-20]. The increased number of studies has driven the need for development of a more user-friendly software for patient-specific EF simulations.

The aim of the paper is to give a presentation of the recent ELMA software improvements of building the brain model, and how these model data can be used as input to preprogrammed FEM simulations and visualizations of the electric field. Furthermore, a novel approach for quick positioning of the DBS lead in a patient-specific manner in the FEM model is presented.

\section{MEthODS}

An overview of the principle modelling and simulation procedure is presented in Fig. 1. Pre- and postoperative images are co-registered and the electrode positions are determined by two points on each electrode artefact in the postoperative images. Electric conductivities are assigned based on tissue classification in the preoperative images. FEM simulations are then performed to determine the electric field which is overlaid on the preoperative images to estimate affected structures.

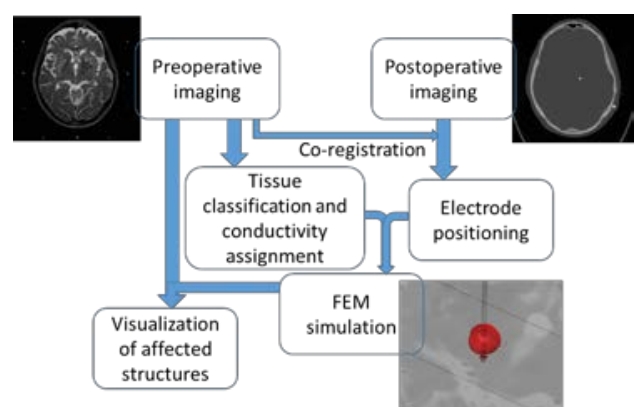

Figure 1. Overview of the patient-specific DBS simulation procedure.

Johannes D. Johansson is with the Department of Biomedical Engineering, Linköping University, 58185 Linköping, Sweden (phone: +46 (0) 10-103 24 64; fax: 013 - 1019 02; e-mail: johannes.johansson@liu.se) Fabiola Alonso and Karin Wårdell are with the Department of Biomedical Engineering, Linköping University, 58185 Linköping, Sweden (e-mail: fabiola.alonso@liu.se; karin.wardell@liu.se) 


\section{A. Image preparation and input requirement}

For the simulations, a preoperative MRI batch of the brain with stereotactic fiducials is needed. Imaging vary between hospitals and surgical targets but, in general, preoperative MRI is performed with a stereotactic frame (Leksell Stereotactic System ${ }^{\circledR}$, Elekta Instrument AB, Sweden) and the fiducialcontaining indicator box attached to the patient's head. Postoperative MRI or computed tomography (CT) is performed with or without the frame in order to confirm electrode positions and screen for hemorrhages. The surgeon notes the electrode positions in Leksell coordinates from the postoperative image artefacts [21, 22] with one point in the lowest electrode contact and one point (e.g. the fourth contact) higher up along the electrode lead. Medical image software (e.g. the open source 3Dslicer, www.slicer.org) is, when needed, used to straighten the pre- and postoperative images. The preprocessed image sets are exported as .nrrd files which can be read into Matlab (Mathworks, USA) with the nrrdread script [23].

\section{B. ELMA software}

An in-house Matlab GUI called ELMA [17] (current version 3.0) is used to convert the medical images into data files readable by the FEM software Comsol Multiphysics 5 (CM, Comsol AB, Sweden) and to assign the relevant electrical conductivities, $\sigma(\mathrm{S} / \mathrm{m})$, from them. Images are read either directly as DICOM files or as preprocessed Matlab files. The fiducial markers are then marked and used to calculate the image set position $\left[x_{\mathrm{m}}, y_{\mathrm{m}}, z_{\mathrm{m}}\right]$ corresponding to the center point $[100,100,100]$ in the Leksell coordinate system in an image at height $z_{i}$ (Fig. 2). The center $z$-coordinate is calculated as

$$
z_{\mathrm{m}}=z_{\mathrm{i}}+l_{\mathrm{z}}-60(\mathrm{~mm})
$$

The image set is then cropped to the desired cuboid size for the simulation geometry. Finally, the tissue is classified into grey matter (GM), white matter (WM), blood, or cerebrospinal fluid (CSF) according to the image intensity values (Fig. 3). Average intensity values are calculated for each tissue type from regions of interest (ROI) close to the surgical target in at least three slices in the preoperative image set. The classification depends on image modality as the tissue types appear different in T1-, T2-, and proton density (PrD)weighted images. Electric conductivities of the voxels are calculated by interpolation of the conductivity values for the two nearest tissue average intensities in the middle third (Fig. 3). The electric conductivities are calculated from tabulated values [24, 25] weighted with the spectral distribution of the Fourier transform of the pulse shape, $H(\omega)$, according to [26]

$$
\sigma=\frac{\int \sigma(\omega) H(\omega)}{\int H(\omega)} d \omega
$$

with the integral going from 10 to $10^{6} \mathrm{~Hz}$. Since the pulse width is much shorter than the period time, it is the main factor affecting the spectral content while the pulse frequency has a negligible impact on tissue conductivity for settings typically used in DBS. Only the conductivities of GM and WM are noticeably affected by the spectral content while the liquids blood and CSF can be considered to have constant conductivities of 0.7 and $2.0 \mathrm{~S} / \mathrm{m}$ respectively.

\section{FEM modelling and simulation of electric fields}

\section{1) Electrode lead placement}

One or two models of the studied DBS lead are made and placed in a box with the same dimensions as the cropped image set. The complete geometry of the FEM model is generated by aligning the individual lead models (Fig. 4) with two points along the DBS lead from the postoperative images as extracted by the surgeon. First, the Leksell [27] coordinates $\left[x_{\mathrm{L}}, y_{\mathrm{L}}, z_{\mathrm{L}}\right]$, where the $\mathrm{z}$-axis is pointing downwards and the $\mathrm{y}$-axis towards the viewer, are converted into regularly oriented Cartesian coordinates with origin in the lower left corner of the image batch by

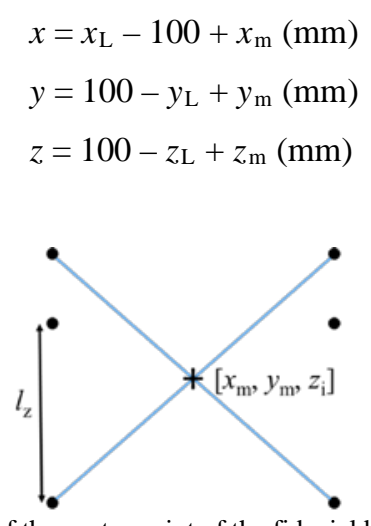

Figure 2. Calculation of the center point of the fiducial box. Due to the Nshape of the fiducials, the $z$-coordinate, $z_{i}$, can be calculated from the distance $l_{z}$ when the image set is properly oriented and be related to the middle point, $z_{\mathrm{m}}$, along the $\mathrm{z}$-axis.

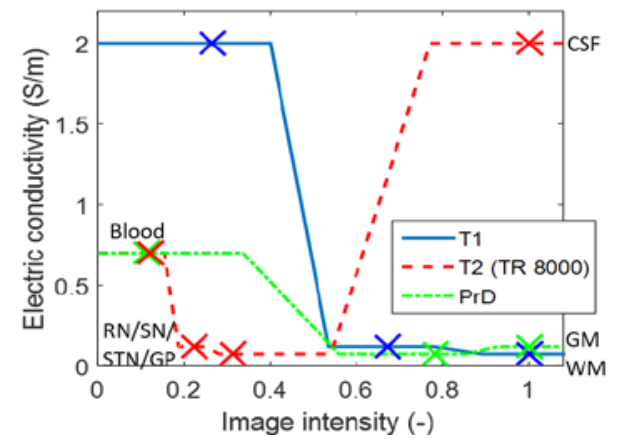

Figure 3. Examples of conductivity values derived from T1-, T2- and PrDweighted MRI versus image intensity normalized with the brightest tissue type. The crosses denote the average intensity values for the different tissue types. At Linköping University hospital, the T2-weighted images are often taken with a repetition time (TR) of 8000 ms. This gives enhanced contrast of the red nucleus $(\mathrm{RN})$, the substantia nigra $(\mathrm{SN})$, the subthalamic nucleus (STN) and the globus pallidus (GP) at the expense of contrast for other grey matter.

The middle point of the lowest electrode contact is then placed at its Cartesian coordinates $\left[x_{0}, y_{0}, z_{0}\right]$ and an axis of rotation orthogonal to the electrode projection in the xy plane is calculated as $\left[y_{0}-y_{1}, x_{1}-x_{0}, 0\right]$ where $\left[x_{1}, y_{1}, z_{1}\right]$ is the Cartesian coordinate higher up the electrode lead (Fig. 4). Alternatively, $\left[x_{0}, y_{0}, z_{0}\right]$ and $\left[x_{1}, y_{1}, z_{1}\right]$ can be read out in CM if pre- and postoperative images have been co-registered in e.g. FSL (https://fsl.fmrib.ox.ac.uk/fsl/fslwiki/ [28]). The axis of rotation does not need to be normalized to unity in CM. The 
electrode lead model is then rotated around this axis by an angle $\varphi$ according to equation (4).

$$
\varphi=\cos ^{-1}\left(\frac{z_{1}-z_{0}}{\sqrt{\left(x_{1}-x_{0}\right)^{2}+\left(y_{1}-y_{0}\right)^{2}+\left(z_{1}-z_{0}\right)^{2}}}\right)
$$
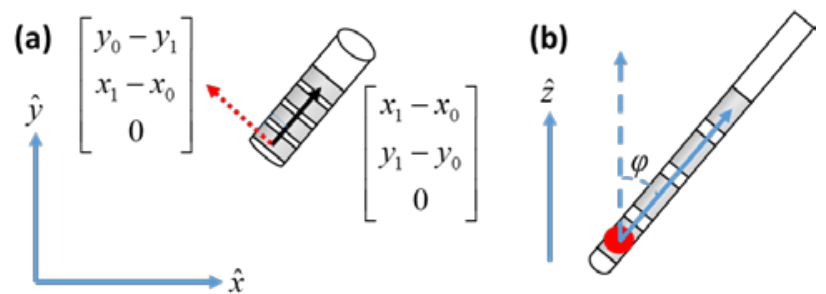

Figure 4. The electrode lead is placed in the model by the coordinates of the lowest lead $\left(x_{0}, y_{0}, z_{0}\right)$ and a point higher up on the lead $\left(x_{1}, y_{1}, z_{1}\right)$. The axis of rotation (left: dotted red arrow, right: red circle) is orthogonal to the tilt direction (black arrow) in the xy plane, i.e. the axial plane.

2) Physical model and boundary conditions

The electric field magnitude, $\mathrm{EF}=|-\nabla V|(\mathrm{V} / \mathrm{m})$, is calculated by the equation for steady currents [29]

$$
\nabla \cdot(\sigma \nabla V)=0 \quad\left(\mathrm{~A} / \mathrm{m}^{3}\right)
$$

where $V$ is the electric potential (V). The electric conductivity, $\sigma$, is loaded as an interpolation function of space (x, y, z) from the brain conductivity file generated by ELMA. Depending on control mode and configuration (monopolar or bipolar), the active electrode surfaces are assigned the clinically used electric potential or current while the outer boundaries of the box are set to ground $(V=0 \mathrm{~V})$. The plastic surfaces between the contacts are set to electric insulation

$$
\mathbf{n} \cdot \nabla V=0(\mathrm{~V} / \mathrm{m})
$$

where $\mathbf{n}$ is the surface normal vector (-). The inactive contacts are set to floating potential with a constant electric potential for each contact

$$
\left[\begin{array}{c}
V \equiv \text { constant }(\mathrm{V}) \\
\int \mathbf{n} \cdot \sigma \nabla V d S=0(\mathrm{~A}) .
\end{array}\right.
$$

\section{3) Visualization}

The EF required to activate axons is dependent on the pulse width and has been estimated to $0.2 \mathrm{~V} / \mathrm{mm}$ at the standard pulse width of $60 \mu$ s down to $0.1 \mathrm{~V} / \mathrm{mm}$ at very long pulse widths of $450 \mu$ s [30]. These isolevels are plotted in slices overlaid on the preoperative MRI images, allowing for identification of included structures of importance.

\section{Clinical examples}

Two simulation examples based on T2-weighted preoperative images from an ET patient with a DBS lead (Model 3389, Medtronic Inc., USA) were set up. The study was approved by the regional ethical board in Linköping (Dnr. 2012/43431) and the patient gave written informed consent. In this particular image set, the T2 sequence used was optimized to visualize the subthalamic nucleus (STN) and globus pallidus (GP), sacrificing contrast between other grey matter and white matter. In the first case, simulations were based on the true patient-specific placement of the lead in the left zona incerta (Zi). In the second simulation example the lead was moved in the brain model to a region close to a pair of CSF-filled cysts in the GP. The second highest contact (contact 2) was used for monopolar stimulation using $3 \mathrm{~V}$. The patient-specific pulse width of $60 \mu$ s and frequency of $130 \mathrm{~Hz}$ were considered when selecting the electrical conductivity values. For the FEM simulations, approximately 800000 tetrahedral elements were used with the elements being smaller close to the electrode surfaces. Simulation times were approximately 2 minutes with the conjugate gradients solver.

\section{RESULTS}

Classification of the tissue when the lead is placed in $\mathrm{Zi}$ is presented in Fig. 5 and visualization of EF isolevel of $0.2 \mathrm{~V} / \mathrm{mm}$ superimposed on the MRI is presented in Fig. 6a.
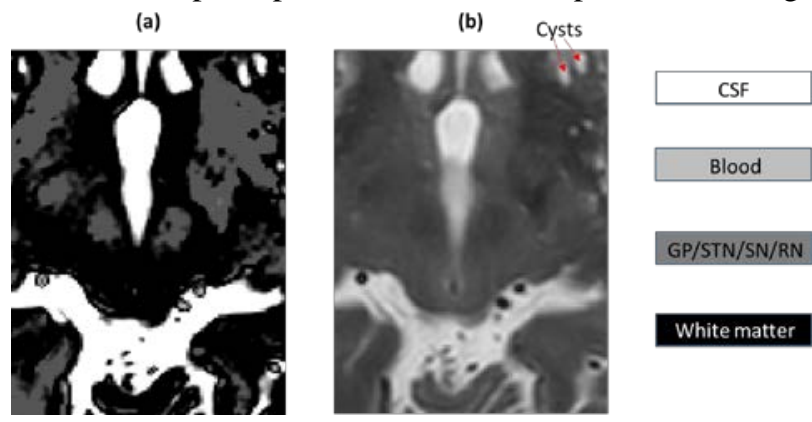

Figure 5. Example of tissue classification (a) from a T2-weighted image set (b). The T2 sequence used here had been optimized to visualize the STN and the GP. It does not provide contrast between the grey matter putamen and the surrounding white matter but a large amount of cysts can be seen in the putamen, e.g. the two white spots in the upper right corner of the images.

For the GP simulation, the cyst image intensity was set to be the one representative of CSF as these small cysts otherwise would have been mostly classified as white matter due to the partial volume effect. It can be seen that the higher electric conductivity of the CSF in the cyst distorts the EF (Fig. 6b). (a)

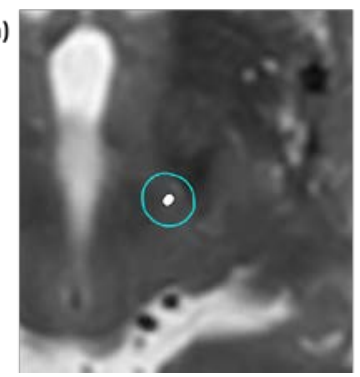

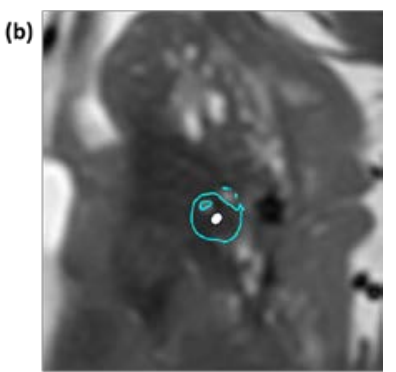

Figure 6. (a) Example of stimulation in the Zi. (b) Example of stimulation near a cyst. The $0.2 \mathrm{~V} / \mathrm{mm}$ cyan isocontour corresponds to the volume estimated to be affected by the DBS.

\section{DISCUSSION}

In this paper, an improved version of the Linköping method for creating patient-specific brain models and 
performing electric field simulations around DBS leads is presented. The software ELMA has been developed for quick generation of conductivity brain models and the lead placement in the simulation software has been made more user-friendly. Simulations using the improved concept are exemplified by DBS leads in brain areas with significant electrical conductivity variations i.e. with and without CSF cysts.

Equation (4) allows for fast alignment of the electrode from two points, either from Leksell coordinates obtained by the surgeons in the surgical planning system or from a coregistered postoperative image set. In our previous studies [10, $19,31]$, the modelled DBS lead had been manually aligned to the postoperative electrode artefact in Comsol Multiphysics. Before, this was a rather time-consuming process as a new simulation had to be done after each lead movement in order to verify the positions of the lead. The position of the electrode leads compared to the postoperative artefacts have been studied in phantom models for CT by Hemm et al. [21] and for MRI by Pollo et al. [22]. In both cases the artefact begins approximately $2 \mathrm{~mm}$ beneath the center of the lowest contact.

The ELMA GUI is a stand-alone application using Matlab's application compiler. It is built as modules that can be extended and updated when new features need to be implemented. We are presently also working on similar applications of patient-specific DBS EF simulations in Comsol Multiphysics. Here, the aim is to have a set of applications including for instance various DBS lead designs and stimulation modes, which can be visualized for a specific isolevel of the EF around the active contacts. An application compiler has recently been made for $\mathrm{CM}$ and we plan to also make our CM applications publicly available as stand-alone applications.

The EF threshold levels of $0.2 \mathrm{~V} / \mathrm{mm}$ at $60 \mu$ s pulse width down to $0.1 \mathrm{~V} / \mathrm{mm}$ at $450 \mu \mathrm{s}$ pulse width have been found to be consistent with clinical experimental studies [7, 8, 13, 30, 32]. It is also possible to export the complete electric potential field, $V$, from the CM simulations in case more complex simulations of individual axons are desired, such as used in e.g. [33], [12] and [32]. It has been estimated from such simulations that the $0.2 \mathrm{~V} / \mathrm{mm}$ threshold level at $60 \mu$ s pulse widths corresponds well to activation of axons with a diameter of around $3 \mu \mathrm{m}$ when using a Matlab-based single cable model [32]. This in turn has been estimated to be approximately corresponding to the commonly used $5.7 \mu \mathrm{m}$ axon diameter [12] in the more complex NEURON ${ }^{\circledR}$ double axon model (https://www.neuron.yale.edu/neuron/) [33]. These are among the larger axon diameters in the brain [34].

Only one image modality for the tissue classification was used in the examples in this paper. It should, however, be noted that all image sequences have different strengths and weaknesses: Proton density images have poor contrast between CSF and grey matter and T1 images have poor contrast for blood (Fig. 3). The T2 imaging protocol used in Linköping give poor contrast between grey and white matter but enhance structures like the STN and RN which are considerably darker, a desirable feature when placing the electrode in the $\mathrm{Zi}$ between these nuclei. It can be beneficial to combine image modalities for better classification when all four tissue types are present in the region of interest. For example, in a previous study, PrD and T1 spoiled gradient echo images were fused into a 3D image volume in order to be able to classify both CSF, white matter, grey matter and blood when building the brain model in ELMA [26].

As can be seen in Fig. 5, the tissue classification has some limitations in terms of resolving the tissue types. For example, there may be small cysts that are not bright enough to be classified as CSF by ELMA when the larger CSF volume of the ventricles is used for characteristic CSF MRI intensity. For this reason, it is of great interest to have good resolution in the images so that the intensities don't smear too much between the voxels, especially as the highly electrically conducting CSF is the tissue type that will distort the EF most compared to a homogeneous tissue model. This is clearly seen in Fig. 6b where the cysts themselves have been used for characteristic CSF MRI intensity. The tissue classification in ELMA is almost instantaneous, which is a benefit when tweaking the parameters for cyst classification. Common tissue classifiers such as FSL FAST and FreeSurfer may take up to 30 minutes or a whole day respectively. Apart from uncertainties in tissue classification, there is also variability of the electric conductivity within the same tissue type. Gabriel et al. [35] mention a spread of their measurement values of $\pm 15-25 \%$. Furthermore, the electric impedance of the brain tissue has been found to change during stimulation [36] and due to edema and gliosis [37]. Also, the tissue in this paper has been assumed to have isotropic electric conductivity while white matter in reality has anisotropic conductivity. An image modality of interest here is diffusion tensor imaging (DTI) which allows estimation of the anisotropy of the electric conductivity rather than just scalar values from the tissue classification and also account for in-tissue variability [18]. Additionally, DTI can be used for tracking fibers between brain structures in relation to DBS. Coenen et al. [38], have presented a simplified electric simulation strategy together with fiber tractography. As a next step the patient-specific simulations will be combined with processed DTI for the combined visualization of white matter fiber tracts together with the patient anatomy [39].

\section{CONCLUSION}

In conclusion, electric field simulations around active DBS contacts have been made more user-friendly with the improved ELMA software for brain model creation, and the direct positioning of the leads in the FEM simulation program allowing faster and simplified patient-specific EF simulations. ELMA and CM applications will be available for download at:

\section{https://liu.se/en/research/dbs}

\section{ACKNOWLEDGMENT}

Older versions of ELMA have initially been developed by Elin Diczfalusy Parker and Mattias Åström.

\section{REFERENCES}

[1] A. L. Benabid et al., "Long-term suppression of tremor by chronic stimulation of the ventral intermediate thalamic nucleus," Lancet, vol. 337, no. 8738, pp. 403-6, Feb 161991.

[2] J. K. Krauss, T. Pohle, S. Weber, C. Ozdoba, and J. M. Burgunder, "Bilateral stimulation of globus pallidus internus for treatment of cervical dystonia," Lancet, vol. 354, no. 9181, pp. 837-8, Sep 04 1999. 
[3] S. Hemm and K. Wårdell, "Stereotactic implantation of deep brain stimulation electrodes: a review of technical systems, methods and emerging tools," Med Biol Eng Comput, vol. 48, no. 7, pp. 611-24, Jul 2010.

[4] B. J. Nuttin et al., "Long-term electrical capsular stimulation in patients with obsessive-compulsive disorder," Neurosurgery, vol. 52, no. 6, pp. 1263-72; discussion 1272-4, Jun 2003.

[5] H. S. Mayberg et al., "Deep brain stimulation for treatmentresistant depression," Neuron, vol. 45, no. 5, pp. 651-60, Mar 03 2005.

[6] V. Vandewalle, C. van der Linden, H. J. Groenewegen, and J. Caemaert, "Stereotactic treatment of Gilles de la Tourette syndrome by high frequency stimulation of thalamus," Lancet, vol. 353, no. 9154, p. 724, Feb 271999.

[7] A. M. Kuncel, S. E. Cooper, and W. M. Grill, "A method to estimate the spatial extent of activation in thalamic deep brain stimulation," Clin Neurophysiol, vol. 119, no. 9, pp. 2148-58, Sep 2008.

[8] M. Rizzone et al., "Deep brain stimulation of the subthalamic nucleus in Parkinson's disease: effects of variation in stimulation parameters," Journal of neurology, neurosurgery, and psychiatry, vol. 71, no. 2, pp. 215-9, Aug 2001.

[9] M. Åström et al., "Patient-Specific Model-Based Investigation of Speech Intelligibility and Movement during Deep Brain Stimulation," (in English), Stereot Funct Neuros, vol. 88, no. 4, pp. 224-233, 2010.

[10] S. Hemm et al., "Patient-Specific Electric Field Simulations and Acceleration Measurements for Objective Analysis of Intraoperative Stimulation Tests in the Thalamus," Frontiers in human neuroscience, vol. 10, p. 577, 2016.

[11] L. Akbarian-Tefaghi et al., "Refining the Deep Brain Stimulation target within the Limbic Globus Pallidus internus for Tourette syndrome " Stereot Funct Neuros, vol. 95, no. 4, pp. 251-258, 2017.

[12] C. C. McIntyre, S. Mori, D. L. Sherman, N. V. Thakor, and J. L. Vitek, "Electric field and stimulating influence generated by deep brain stimulation of the subthalamic nucleus," Clin Neurophysiol, vol. 115, no. 3, pp. 589-95, Mar 2004.

[13] S. Hemm, G. Mennessier, N. Vayssiere, L. Cif, and P. Coubes, "Co-registration of stereotactic MRI and isofieldlines during deep brain stimulation," Brain research bulletin, vol. 68, no. 1-2, pp. 59-61, Dec 152005.

[14] M. Åström, J. D. Johansson, M. I. Hariz, O. Eriksson, and K. Wårdell, "The effect of cystic cavities on deep brain stimulation in the basal ganglia: a simulation-based study," Journal of neural engineering, vol. 3, no. 2, pp. 132-8, Jun 2006.

[15] C. R. Butson, S. E. Cooper, J. M. Henderson, and C. C. McIntyre, "Patient-specific analysis of the volume of tissue activated during deep brain stimulation," NeuroImage, vol. 34, no. 2, pp. 661-70, Jan 152007.

[16] M. Åström, L. U. Zrinzo, S. Tisch, E. Tripoliti, M. I. Hariz, and K. Wårdell, "Method for patient-specific finite element modeling and simulation of deep brain stimulation," Med Biol Eng Comput, vol. 47, no. 1, pp. 21-8, Jan 2009.

[17] K. Wårdell, E. Diczfalusy, and M. Åström, "Patient-Specific Modeling and Simulation of Deep Brain Stimulation," in PatientSpecific Modeling in Tomorrow's Medicine, Studies in mechanobiology, tissue engineering and biomaterials, No. 9, 2011, pp. 357-378.

[18] M. Åström, J. J. Lemaire, and K. Wårdell, "Influence of heterogeneous and anisotropic tissue conductivity on electric field distribution in deep brain stimulation," Med Biol Eng Comput, vol. 50, no. 1, pp. 23-32, Jan 2012.

[19] F. Alonso, M. A. Latorre, N. Göransson, P. Zsigmond, and K. Wårdell, "Investigation into Deep Brain Stimulation Lead Designs: A Patient-Specific Simulation Study," (in English), Brain Sci, vol. 6, no. 3, Sep 2016.

[20] H. Akram et al., "Subthalamic deep brain stimulation sweet spots and hyperdirect cortical connectivity in Parkinson's disease," NeuroImage, vol. 158, pp. 332-345, Sep 2017.

[21] S. Hemm et al., "Contact position analysis of deep brain stimulation electrodes on post-operative CT images," (in English), Acta Neurochir, vol. 151, no. 7, pp. 823-829, Jul 2009.
[22] C. Pollo, J. G. Villemure, F. Vingerhoets, J. Ghika, P. Maeder, and R. Meuli, "Magnetic resonance artifact induced by the electrode Activa 3389: an in vitro and in vivo study," (in English), Acta Neurochir, vol. 146, no. 2, pp. 161-164, Feb 2004.

[23] J. Mather. (2012). nrrdread [Online]. Available: http://www.mathworks.com/matlabcentral/fileexchange/34653nrrd-format-file-reader/content/nrrdread.m.

[24] S. Gabriel, R. W. Lau, and C. Gabriel, "The dielectric properties of biological tissues: III. Parametric models for the dielectric spectrum of tissues," Physics in medicine and biology, vol. 41, no. 11, pp. 2271-93, Nov 1996.

[25] D. Andreucetti, R. Fossi, and C. Petrucci. (1997). An Internet resource for the calculation of the dielectric properties of body tissues in the frequency range $10 \mathrm{~Hz}-100 \mathrm{GHz}$ [Online]. Available: http://niremf.ifac.cnr.it/tissprop/.

[26] K. Wårdell, L. Zrinzo, M. Hariz, and M. Andersson, "PatientSpecific Brain Modelling for Deep Brain Stimulation Simulations," (in English), I IEEE EMBS C Neur E, pp. 148-151, 2013.

[27] L. D. Lunsford, D. Kondziolka, and D. Leksell, "Leksell Stereotactic Apparatus," in Textbook of Stereotactic and Functional Neurosurgery, A. M. Lozano, P. L. Gildenberg, and R. R. Tasker, Eds. Berlin, Heidelberg: Springer Berlin Heidelberg, 2009, pp. 469-485.

[28] M. Jenkinson, P. Bannister, M. Brady, and S. Smith, "Improved optimization for the robust and accurate linear registration and motion correction of brain images," (in English), NeuroImage, vol. 17, no. 2, pp. 825-841, Oct 2002.

[29] D. K. Cheng, Field and wave electromagnetics, 2 ed. AddisonWesley, 1989.

[30] J. Johansson, F. Alonso, and K. Wårdell, "Modelling details for electric field simulations of deep brain stimulation," in World Congress on Medical Physics \& Biomedical Engineering, Prague, 2018, vol. 68, no. 1: Springer, pp. 645-648.

[31] K. Wårdell et al., "Deep brain stimulation of the pallidum internum for Gilles de la Tourette syndrome: a patient-specific model-based simulation study of the electric field," Neuromodulation : journal of the International Neuromodulation Society, vol. 18, no. 2, pp. 90-6, Feb 2015.

[32] M. Åström, E. Diczfalusy, H. Martens, and K. Wårdell, "Relationship between Neural Activation and Electric Field Distribution during Deep Brain Stimulation," (in English), IEEE T Bio-Med Eng, vol. 62, no. 2, pp. 664-672, Feb 2015.

[33] M. Latorre and K. Wårdell, "A comparison between single and double cable neuron models applicable to deep brain stimulation," Biomedical Physics \& Engineering Express, 2019.

[34] D. Liewald, R. Miller, N. Logothetis, H. J. Wagner, and A. Schuz "Distribution of axon diameters in cortical white matter: an electron-microscopic study on three human brains and a macaque," (in English), Biol Cybern, vol. 108, no. 5, pp. 541-557, Oct 2014.

[35] S. Gabriel, R. W. Lau, and C. Gabriel, "The dielectric properties of biological tissues: II. Measurements in the frequency range 10 $\mathrm{Hz}$ to $20 \mathrm{GHz}$," Physics in medicine and biology, vol. 41, no. 11, pp. 2251-69, Nov 1996.

[36] S. Hemm et al., "Evolution of brain impedance in dystonic patients treated by GPI electrical stimulation," Neuromodulation : journal of the International Neuromodulation Society, vol. 7, no. 2, pp. 67-75, Apr 2004.

[37] C. Lungu et al., "Temporal macrodynamics and microdynamics of the postoperative impedance at the tissue-electrode interface in deep brain stimulation patients," Journal of neurology, neurosurgery, and psychiatry, vol. 85, no. 7, pp. 816-9, Jul 2014.

[38] V. A. Coenen, N. Allert, S. Paus, M. Kronenburger, H. Urbach, and B. Madler, "Modulation of the cerebello-thalamo-cortical network in thalamic deep brain stimulation for tremor: a diffusion tensor imaging study," Neurosurgery, vol. 75, no. 6, pp. 657-69; discussion 669-70, Dec 2014.

[39] T. Nordin, P. Zsigmond, S. Pujol, C.-F. Westin, and K. Wårdell, "Deep brain stimulation: Patient-specific electrical field simulation and tractography," in ESSFN XXIII congress, Edinburgh, 2018. 